\title{
Mega Watt Security Assessment of Power Systems
}

\author{
Amit kumar Chowdhury ${ }^{1}$, Surajit Mondal ${ }^{2}$,Mainak Mukherjee ${ }^{3}$, \\ Pabitra kumar Biswas ${ }^{4}$ \\ ${ }^{1} \mathrm{M} . \mathrm{E}$,Electrical Engineering Dept, IIEST , Shibpur \\ ${ }^{2}$ Research Scholar, University of Petroleum \& Energy Studies, Dehradun \\ ${ }^{3}$ M.Tech(Energy Systems), University of Petroleum \& Energy Studies, Dehradun \\ ${ }^{4}$ H.O.D.,Electrical Engineering Dept, NIT Mizoram \\ E-mail address: amitchowdhury91@gmail.com
}

Keywords: DC load Flow, Fast decoupled load flow, performance index, Masking Problem.

\begin{abstract}
This paper deals with the security aspects of power system by evaluating the severity of transmission line outage. MW security assessment is made by determining the power flow in the line using load flow for each contingency. The severity of contingency is measured using a scalar index called performance index (PI). DC load flow and Fast Decoupled load flow are used as approximate and exact load flow methods for MW security assessment respectively. Contingency analysis is carried out and ranked lists in the decreasing order of severity based on PI values are prepared for standard test systems. The severity of line is evaluated and compared using these load flow methods. A new method is proposed to avoid Masking problems in MW security assessment. Security analysis is made on standard test systems such as 5, 6, IEEE 14 and IEEE 30 bus systems under present study.
\end{abstract}

\section{INTRODUCTION}

The production of electric energy is the backbone of growth of the National economy. The social and economic consequences of a major power supply interruption are so great that every effort must be made to reduce the impact of such a disturbance.

Reliable operation of power grid is therefore crucially important. Demand in electric power in a developing country increases almost exponentially every year. The existing generation and transmission system must operate very near to their capacity. During operation, power systems are routinely affected by unpredictable faults and failures.

Therefore power system should be operated in such a way so that it should be able to withstand such disturbances. Hence, security aspect must be investigated with intimate attention to the problem so that a reliable operation can be ensured to the consumers. The North American Electric Reliability Council defined reliability of a power system with two components namely adequacy and security.

Adequacy is the ability to supply energy in order to meet the load demand. Security is the ability of a system to withstand sudden disturbances without violating any operational constraints. Disturbances may be short circuits, change in network configuration due to faults, loss of system components, sudden increase in load etc.

\section{On-Line Security Analysis}

In an on-line environment security analysis differs from that at planning stage. In the planning stage, security analysis is carried out to test the ability of the system to withstand unexpected losses of certain system components. For the time varying real world operation, the system may experience severe insecurity from the operators' perspective. For example, a variety of elements may undergo unscheduled shutdown, repair etc or bad weather condition which may be different from those studied in planning stage. Therefore an operator should be well equipped to cope up with any unplanned causalities to any operating equipment. In an on-line security analysis about 15 minutes time period is normally recommended for analysis and reporting. 


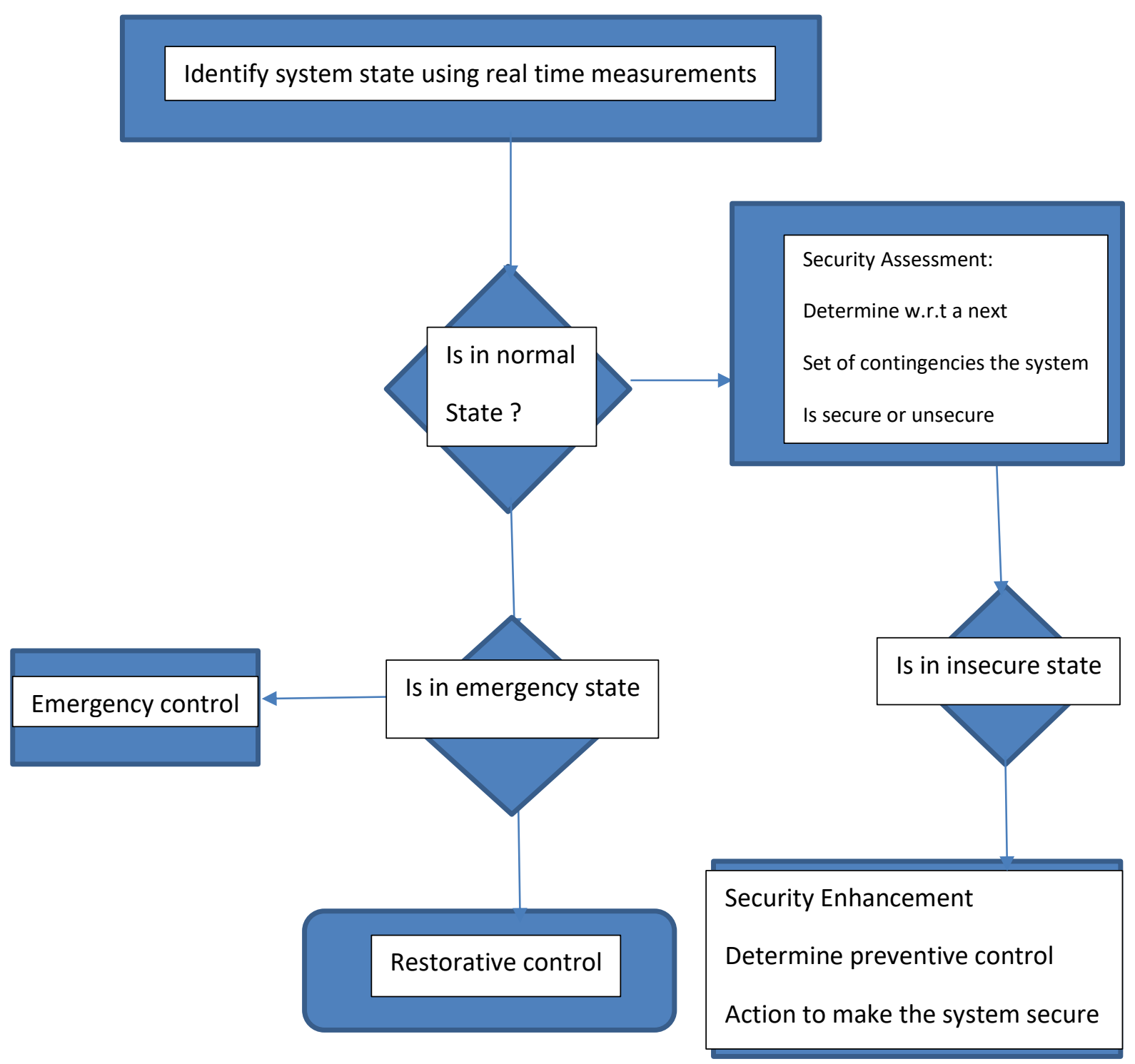

\section{TOOLS AND TECHNIQUES FOR SECURITY ANALYSIS}

The basic tool for Steady State Security Analysis is the Load flow. It is impractical to study all the possibilities of circuit outages by full ac load flow techniques due to time constraints in online environment. To assess steady state active power condition quickly, Linearized or DC load flow (DCLF) model is used. This approach gives only real power solution. After that Fast decoupled load Flow (FDLF) comes into play.FDLF fulfills two basic requirements i.e., speed and accuracy in an on-line computation. Steady State Security Analysis deals with Contingency Analysis, Ranking, Selection and Evaluation.

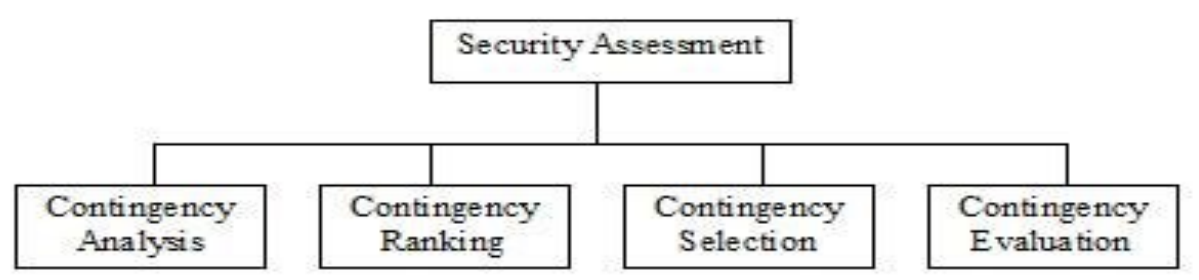

\section{CONTINGENCY ANALYSIS}

Contingency analysis is a software application run in an energy management system to give the operator an indication of what might happen to the power System in the event of an unplanned 
(unscheduled) equipment outage. That is, the contingency analysis application allows the operator to ask what if

Questions such as: What will be the state of the system if we have an outage on part of a major $500 \mathrm{KV}$ transmission system? The answer to this question might be that the system power flows and voltages will read just and remain within acceptable limits, or the answer might be that severe overloads and/or under-voltages will occur such that the system survival will exists or not. An overload itself can damage the transmission and the generating equipment if it is severe enough and exists for a long period.

\section{CONTINGENCY RANKING AND SELECTION [1]}

Contingency Ranking in descending order is obtained according to the values of a scalar index, normally called Performance Index-(PI). PI is a measure of system-wide severity or impact of a contingency event in the system.

Severe contingencies are then chosen from either the ranked lists starting from the top and going down the list until a predefined stopping criteria is reached. The process of choosing a subset containing only the severe contingencies is contingency selection.

\section{Performance Index of a Line}

$$
\mathrm{PI}=\sum_{l=1}^{N L}\left(\frac{\mathrm{WL}}{2 \mathrm{n}}\right)\left(\frac{\mathrm{p}^{\mathrm{l}}}{\mathrm{p}^{\lim }}\right)^{2 \mathrm{n}}
$$

where,

$\mathrm{P}^{1}=$ The MW flow of line 1.

$\mathrm{P}^{\mathrm{Lim}}=$ The MW capacity of line

$\mathrm{WL}=$ Real non-negative weighting coefficient.

$\mathrm{NL}=$ total no of lines of the system

The Performance Index PI, contains all line flows normalized to their limits. These normalized flows are raised to an even power (by setting $n=1,2, \ldots$ ) thus the use of absolute magnitude is ensured. This index has a small value when all line flows are within their limits and higher value in violating cases. Thus it provides a good measure of severity of line overloads for a given state of power system.

\section{Comparison of PI between DCLF and FDLF methods}

The entire MW ranking of IEEE6 Bus system obtained by DCLF and FDLF methods are compared. Since conventional method of PIMW is used, masking effect will exist in both the methods. The ranking obtained by FDLF method is compared with that of DCLF method. This shifting of a particular line from the exact ranking is denoted by upward shift (US) or by downward shift (DS) shift. The positional shift (PS) is expressed by numerical value 's'. If a line is found to be appeared in the same position as that of FDLF method, then the line is said to be ranked correctly which is represented by correct ranking (CR). Comparison of ranking of IEEE6 bus system is given in the following table.

\begin{tabular}{|l|l|l|l|}
\hline Rank & FDLF & DCLF & PS \\
\hline 1 & 2 & 2 & CR \\
\hline 2 & 3 & 3 & CR \\
\hline 3 & 9 & 7 & D1 \\
\hline 4 & 7 & 9 & U1 \\
\hline 5 & 5 & 8 & D1 \\
\hline
\end{tabular}




\begin{tabular}{|l|l|l|l|}
\hline 6 & 8 & 5 & $\mathrm{U} 1$ \\
\hline 7 & 6 & 1 & $\mathrm{D} 1$ \\
\hline 8 & 1 & 6 & $\mathrm{U} 1$ \\
\hline 9 & 11 & 11 & $\mathrm{CR}$ \\
\hline 10 & 10 & 4 & $\mathrm{D} 1$ \\
\hline 11 & 4 & 10 & $\mathrm{U} 1$ \\
\hline
\end{tabular}

\section{Selection of Contingencies [1]}

Entire list of contingencies are avoided for accurate load flow analysis. It is therefore required to select only a prescribed subset of contingencies. The subset should contain critical cases which effects the security of the system. The noncritical cases increase the computation burden, while a critical case being dangerous will effect system operation. Sometimes, a very few un-critical cases are tolerated when there is no alternative but even a single case of critical contingency should never be kept outside of the selection list. Therefore, the contingency selection algorithm should be capable of capturing all important cases.

In general, three ways of contingency selections are normally adopted.

\section{Absolute Fixed list:}

Select a fixed number, say ' $N$ ' number of contingencies starting from the topmost of the list irrespective of size of the system. This process of selection may however miss many critical cases if $\mathrm{N}$ is not sufficiently large.

\section{System dependent Fixed list:}

Select a predefined set, say $\mathrm{k} \%$ of the contingencies starting from the topmost of the ranked list. The value of $\mathrm{k}$ may be 10,15 or more. Therefore, the number of contingencies varies with the system size. To capture all the harmful cases this selection process is not only highly dependent on the accuracy of the fast approximate contingency analysis algorithm but also on the effectiveness of the ranking algorithm. 'Masking problem' may still remain in this selection process.

\section{Variable list:}

Start accurate full ac load flow analysis beginning with the topmost one and going down the ranked list till a 'no problem case' case arises. This procedure may also miss some cases if the selection is stopped at some point. Therefore, it is suggested to go on analyzing some more cases moving down the list till $\mathrm{M}$ successive cases are found to be 'noncritical'. The value of $\mathrm{M}$ may be chosen as 2, 3 or more. The suitability of this approach is mostly dependent on the accuracy of the ranking algorithm. Computational burden may be increased in analyzing many uncritical cases in this selection algorithm.

For present analysis "system dependent fixed list" procedure is adopted for line contingency selection.

\begin{tabular}{|c|c|c|}
\hline Bus System & Total Numbers of Lines & Selected Lines $(20 \%)$ \\
\hline IEEE-5 BUS & 7 & $L_{1} ; L_{5}$ \\
\hline IEEE-6 BUS & 11 & $L_{2} ; L_{3} ; L_{9}$ \\
\hline IEEE-14BUS & 20 & $L_{1} ; L_{2} ; L_{8} ; L_{4}$ \\
\hline IEEE-30BUS & 41 & $L_{1} ; L_{5} ; L_{6} ; L_{15} ; L_{36}$ \\
\hline
\end{tabular}


MW Limit Violation Cases using FDLF

\begin{tabular}{|c|c|c|c|c|c|}
\hline Test System & $\begin{array}{c}\text { Number of } \\
\text { Violation } \\
\text { cases }\end{array}$ & $\begin{array}{l}\text { Tripped } \\
\text { Line NO. }\end{array}$ & Rank & $\begin{array}{c}\text { Overloaded } \\
\text { Line }\end{array}$ & $\begin{array}{r}\text { Loading } \\
\%\end{array}$ \\
\hline IEEE 5-Bus & 1 & $L_{1}$ & 1 & 2 & 1.7977 \\
\hline IEEE 6-Bus & NIL & - & - & - & - \\
\hline \multirow[t]{8}{*}{$\begin{array}{l}\text { IEEE 14- } \\
\text { Bus }\end{array}$} & \multirow[t]{8}{*}{8} & \multirow[t]{2}{*}{${ }^{L} 1$} & \multirow[b]{2}{*}{2} & $\begin{array}{l}4 \\
7\end{array}$ & $\begin{array}{l}3.1710 \\
1.3844\end{array}$ \\
\hline & & & & $\begin{array}{l}3 \\
4 \\
6 \\
7\end{array}$ & $\begin{array}{l}1.1621 \\
1.1606 \\
1.1963 \\
1.0508\end{array}$ \\
\hline & & $L_{3}$ & 5 & $\begin{array}{l}2 \\
4 \\
7\end{array}$ & $\begin{array}{l}1.0955 \\
1.1203 \\
1.0045\end{array}$ \\
\hline & & ${ }^{L} 4$ & 4 & $\begin{array}{l}1 \\
2 \\
3 \\
5\end{array}$ & $\begin{array}{l}1.4519 \\
1.0555 \\
1.0342 \\
1.0075\end{array}$ \\
\hline & & \multirow{3}{*}{$\begin{array}{l}L_{5} \\
L_{6} \\
L_{7}\end{array}$} & \multirow{3}{*}{$\begin{array}{c}8 \\
12 \\
6\end{array}$} & 4 & 1.1105 \\
\hline & & & & 2 & 1.1951 \\
\hline & & & & $\begin{array}{l}1 \\
2 \\
3\end{array}$ & $\begin{array}{l}1.0768 \\
1.0824 \\
1.1055\end{array}$ \\
\hline & & $L_{8}$ & 3 & 7 & 1.0313 \\
\hline \multirow[t]{2}{*}{$\begin{array}{c}\text { IEEE 30- } \\
\text { Bus }\end{array}$} & \multirow[t]{2}{*}{2} & \multirow[t]{2}{*}{${ }^{L} 1$} & \multirow[t]{2}{*}{1} & $\begin{array}{l}2 \\
4\end{array}$ & $\begin{array}{l}1.7855 \\
1.5060\end{array}$ \\
\hline & & & & 5 & 1.2057 \\
\hline
\end{tabular}

\section{Alleviation of Line Overloads [4]}

A large power transmission network is accompanied with continuous changes of its operating conditions. Abnormal situation prevails in the system when these changes are very large. The changes include outage of any line or generator, tripping of any system component or power exchange with selected neighboring networks. . So it is necessary to determine the approximate effect of these changes over the whole network. The network change may cause MW-limit violation in lines. These results have harmful effects on power system.

The controlling devices are normally installed in the system to remove these limit violation problems. It helps to bring back the line flows within limits. There are various controlling devices 
that can be remotely controlled by an operator (dispatcher) to adjust real power flow directly.

- Generator real power set point.

- Pumped storage input or output.

- Load shedding.

When the first two devices (or actions) are not available (not possible) then the load shedding method is adopted. This method is undesirable because the service to consumers is interrupted. So this is mainly an emergency control to be used when demand exceeds generator capacity.

\section{MASKING PROBLEM IN MW SECURITY ASSESSMENT [3]}

The lack of discrimination in which the performance index for a case with many small violations may be comparable in value to the index for a case with one huge violation, is known as Masking Effect. In other words it may be stated as the problem with the misclassification of contingency case obtained by ranking algorithm in such way that a non-critical contingency ranked as critical or a critical contingency ranked as non-critical. Miss-ranking of contingencies are mainly due to inaccuracies in the model used for computing performance index. Miss-ranking is characterized by errors in the computed order of relative severities of various contingencies. Masking Effect is dangerous when a severe contingency is ranked lower in the ranking list. Selection of harmful contingencies is a big problem for an operator to initiate control actions. Contingency ranking is an important part of security analysis. In this analysis line outages are only considered for contingency analysis. The position of a line in the ranking list indicates the severity of an outage. Hence security assessment depends solely on the accuracy of ranking algorithm.

Simplest and efficient mathematical way to eliminate this problem is to increase the exponent in the formula used to calculate PI of lines.

$$
P I=\sum_{l=1}^{N L}\left(\frac{W L}{2 n}\right)\left(\frac{P l}{P L i m}\right)^{2 n}
$$

Here, ' $n$ ' is the exponent. The accuracy of the conventional method of ranking may be increased by increasing the value of the exponent. As ' $n$ ' increases, loading ratio decreases for lines whose value is less than 100 percent and loading ratio increases for lines whose value is greater than 100 percent. Hence, increment of 'n' may reduce masking effect to some extent. The selection of ranking algorithm to avoid masking problem depends on nature and size of the system. For larger systems, fuzzy logic technique is ideal method for eliminating masking problem.

\section{CONCLUSION}

MW Security Assessment of Power Systems is important to maintain proper functioning of grid. Security assessment should be correct with ideal ranking algorithm. The results of security analysis will help the operators in power system control centers to take preventive control action. The results presented in this paper are based on traditional method of ranking algorithm. Masking effect exists in traditional method of MW security assessment. Ranking algorithms developed from the last decade yield good results for its own system. A unique ranking algorithm is not applicable for all the systems. An ideal power system security assessment can be done using advanced techniques like Artificial Neural Network (ANN), Artificial Intelligence etc 


\section{References}

[1] N. Balu, T. Bertram, A. Bose, V. Brandwajn, G. Cauly, D. Curtice, A. Fouad, L. Fink, M. G. Lauby, B. F. Wollenbarg and J. N. Wrubel. "On-Line Power System Security Analysis". IEEE Proc., Vol. 80, no. 2, Feb., 1992, pp.261-281.

[2] B. Stott, O. Alsac, et al. "Security Analysis and Optimization". Pro-ceedings of the IEEE, Vol. 75, No. 12, Dec, 1987.

[3] G. C. Ejebe and B. F. Wollenberg. "Automatic Contingency Selection.” IEEE Trans., PAS98, no. 1, Jan/Feb., 1979, pp.97-109.

[4] B. Stott and O. Alsac, "Fast Decoupled Load flow". IEEE transactions on PAS, Vol. 93, 1974. 